\title{
Embeddings of totally ordered MV-algebras of bounded cardinality
}

\author{
by \\ Piotr J. Wojciechowski (El Paso, TX)
}

\begin{abstract}
For a given cardinal number $\mathfrak{a}$, we construct a totally ordered MV-algebra $M(\mathfrak{a})$ having the property that every totally ordered MV-algebra of cardinality at most $\mathfrak{a}$ embeds into $M(\mathfrak{a})$. In case $\mathfrak{a}=\aleph_{0}$, the algebra $M(\mathfrak{a})$ is the first known MV-algebra with respect to which the deductive system for the infinitely-valued Lukasiewicz's propositional logic is strongly complete.
\end{abstract}

1. Preliminaries. All necessary information about MV-algebras and lattice-ordered groups can be found in [6] and in [1], respectively. We use two main tools: the Mundici Categorical Equivalence Theorem [10] establishing the equivalence between the category of MV-algebras and the category of unital abelian lattice-ordered groups, and the Hahn Embedding Theorem that allows one to view an abelian totally ordered group as a certain group of real functions ([1, Chapter 3]). Before proceeding to the main result, let us recall these two facts along with the relevant definitions.

If $G$ is an abelian lattice-ordered group ( $\ell$-group) and $u>0$ is an element of $G$, then $u$ is a strong order unit if for any positive element $a \in G$ there exists a positive integer $n$ such that $n u>a$. Another way of phrasing it is to say that the set $G^{+}$of all positive elements of $G$ satisfies $G^{+}=\bigcup_{n=1}^{\infty}[0, n u]$, where for any $a, b \in G$, we define $[a, b]=\{x \in G: a \leq x \leq b\}$. An $\ell$-group with a strong order unit is called unital.

Given any abelian $\ell$-group $G$ and a positive element $u$ in $G$, by $\Gamma(G, u)$ is denoted the MV-algebra $([0, u], \oplus, \neg, 0)$ where the operations are defined by $x \oplus y=(x+y) \wedge u$ and $\neg x=u-x$. Conversely, for every MV-algebra $A$ there exists an abelian $\ell$-group $G$ with a strong order unit $u$ such that $A$ is isomorphic to $\Gamma(G, u)$. Moreover, the morphism $\Gamma$ establishes an equivalence between the two categories (Mundici [10]).

2000 Mathematics Subject Classification: 06D35, 06F20.

Key words and phrases: MV-algebra, lattice-ordered abelian group, Łukasiewicz deductive system. 
Since all our $\ell$-groups here are going to be totally ordered (o-groups), we focus on the relevant embedding theorem for the abelian o-groups. Let $\Lambda$ denote any totally ordered set, and consider real functions from $\Lambda$. For each such function $f$, let the support of $f$ be the set $\operatorname{supp}(f)=\{\lambda \in \Lambda: f(\lambda) \neq 0\}$. The set of all functions whose support satisfies the ascending chain condition (relative to the total order of $\Lambda$ ) forms an abelian o-group if addition is defined pointwise and a function is considered positive if and only if its value at the maximal element of its support is positive. This o-group is called the Hahn o-group on the totally ordered set $\Lambda$, and is denoted by $V(\Lambda, \mathbb{R})$.

The Hahn Embedding Theorem says that every abelian $o$-group embeds into the Hahn group $V(\Lambda, \mathbb{R})$ for some totally ordered set $\Lambda$. Suppose then that $G$ is a subgroup of $V(\Lambda, \mathbb{R})$. We will use the convention that for every $g \in G, g_{\lambda}$ denotes the $\lambda$-component of $g$, i.e. the real value $g$ assumes at $\lambda$. Using the generalized sequence notation for clarity, we identify $g$ with $\left(\ldots, g_{\lambda}, \ldots\right)$.

Finally, if $T$ is an arbitrary totally ordered set with a maximal element $m$, let $\mathbf{1}(T)$ denote the characteristic function of $T$, so that $\mathbf{1}(T): T \rightarrow \mathbb{R}$ and

$$
\mathbf{1}(T)(t)= \begin{cases}1 & \text { if } t=m \\ 0 & \text { if } t<m\end{cases}
$$

2. Result. The following lemma is partially based on [7].

Lemma 2.1. Let $u \in V(\Lambda, \mathbb{R})$ be such that $u_{\mu}>0$ for $\mu$ the maximal element of $\Lambda$. Then there exists an o-group automorphism $\phi$ of $V(\Lambda, \mathbb{R})$ such that $\phi(u)=\mathbf{1}(\Lambda)$.

Proof. For every $g \in V(\Lambda, \mathbb{R})$, we define $\phi(g)$ by specifying its components:

$$
(\phi(g))_{\lambda}= \begin{cases}g_{\lambda}-u_{\lambda} \cdot g_{\mu} / u_{\mu} & \text { if } \lambda \neq \mu, \\ g_{\mu} / u_{\mu} & \text { if } \lambda=\mu .\end{cases}
$$

Since addition is pointwise, it is routine to check that $\phi$ is an o-group homomorphism. Also, $\phi(g)_{\lambda}=0$ for every $\lambda \in \Lambda$ implies that $g_{\lambda}=0$ for every $\lambda$, so $g=0$, and thus $\phi$ is one-to-one. That $\phi(g)$ is an element of $V(\Lambda, \mathbb{R})$ follows from the fact that clearly $\operatorname{supp}(\phi(g)) \subseteq \operatorname{supp}(g) \cup \operatorname{supp}(u)$ and thus $\operatorname{supp}(\phi(g))$ satisfies the ascending chain condition since both $\operatorname{supp}(g)$ and $\operatorname{supp}(u)$ do.

If $f=\left(\ldots, f_{\lambda}, \ldots\right) \in V(\Lambda, \mathbb{R})$, then if we let

$$
g_{\lambda}= \begin{cases}f_{\lambda}+u_{\lambda} \cdot f_{\mu} & \text { if } \lambda \neq \mu, \\ u_{\mu} \cdot f_{\mu} & \text { if } \lambda=\mu,\end{cases}
$$

then for similar reasons as before, $g=\left(\ldots, g_{\lambda}, \ldots\right) \in V(\Lambda, \mathbb{R})$, and $f=\phi(g)$, so $\phi$ is onto $V(\Lambda, \mathbb{R})$. 
Finally,

$$
(\phi(u))_{\lambda}= \begin{cases}u_{\lambda}-u_{\lambda} \cdot u_{\mu} / u_{\mu}=0 & \text { if } \lambda \neq \mu, \\ u_{\mu} / u_{\mu}=1 & \text { if } \lambda=\mu,\end{cases}
$$

so $\phi(u)=\mathbf{1}(\Lambda)$.

COROLlary 2.2. For every abelian o-group $G$ with a strong order unit $u$ there exists a totally ordered set $\Lambda$ with a maximal element such that $G$ embeds into $V(\Lambda, \mathbb{R})$ with u mapped to $\mathbf{1}(\Lambda)$. Moreover, the set $\Lambda$ can be chosen to have the same cardinality as $G$.

Proof. By the Hahn Theorem, $G$ embeds into $V(\Lambda, \mathbb{R})$ for some totally ordered set $\Lambda$. Since $G$ is unital, we can assume that $\Lambda$ has a maximal element $\mu$. Then $u_{\mu}>0$ and the assumptions of Lemma 2.1 are satisfied. Therefore, via the automorphism $\phi, G$ embeds into $V(\Lambda, \mathbb{R})$ so that $u$ is mapped to $\mathbf{1}(\Lambda)$.

In order to prove the cardinality statement, we refer to the construction of the set $\Lambda$ as described in [1, Chapter 3]. In the Hahn group, the set $\Lambda$ consists of the so-called regular subgroups of $G$ (in an $\ell$-group $G$, a subgroup is regular if it is a convex $\ell$-subgroup maximal with respect to missing some element from the group). It is well-known that in an $o$-group, there is precisely one such subgroup per nonzero element (o-groups are special-valued). Therefore we can always find $\Lambda$ such that $|\Lambda| \leq|G|$. In case this inequality is strict, we can extend $\Lambda$ to a totally ordered set of cardinality $|G|$ with a maximal element $\mu$, and the embedding will obviously still hold true.

Lemma 2.3. For every cardinal number $\mathfrak{a}$ there exists a totally ordered set $\widehat{\Delta}(\mathfrak{a})$ with a maximal element such that for every totally ordered set $\Lambda$ with a maximal element $\mu_{\Lambda}$ and of cardinality at most $\mathfrak{a}, V(\Lambda, \mathbb{R})$ embeds into $V(\widehat{\Delta}(\mathfrak{a}), \mathbb{R})$ in such a way that $\mathbf{1}(\Lambda)$ is mapped to $\mathbf{1}(\widehat{\Delta}(\mathfrak{a}))$.

Proof. Let $\Xi$ be an arbitrary set of cardinality $\mathfrak{a}$. Let $\Psi$ be the set of all total orders $<_{\psi}$ on $\Xi$ that have a maximal element. Totally order $\Psi$ any way by some relation $<$. Then consider the disjoint union $\Delta$ of copies of $\Xi$ indexed by the elements of $\Psi$, so that $\Delta=\bigcup_{\psi \in \Psi} \Xi_{\psi}$, where $\Xi_{\psi}=\Xi$ and is ordered by $<_{\psi}$. Now totally order $\Delta$ by $\prec$, where for $\delta, \sigma \in \Delta, \delta \prec \sigma$ if and only if either $\delta \in \Xi_{\psi}$ and $\sigma \in \Xi_{\tau}$ and $\psi<\tau$ (in $\Psi$ ), or for some $\psi \in \Psi$, $\delta, \sigma \in \Xi_{\psi}$ and $\delta<_{\psi} \sigma$ (in $\Xi_{\psi}$ ). It is obvious that this yields a total order.

Finally, let $\widehat{\Delta}(\mathfrak{a})=\Delta \cup\{\mu\}$ and let $\mu$ be the maximal element of $\widehat{\Delta}(\mathfrak{a})$ in the extended order.

Let $\phi: \Lambda \rightarrow \Xi$ be a set embedding granted by the cardinality condition $|\Lambda| \leq \mathfrak{a}$. Let us choose a total order $\psi$ on $\Xi$ such that for all $\alpha, \beta \in \Lambda$, if $\alpha<_{\Lambda} \beta$, then $\phi(\alpha)<_{\psi} \phi(\beta)$. This way $\phi$ becomes an order embedding of the totally ordered set $\Lambda$ into $\Xi_{\psi}$. Let now $\widehat{\phi}: \Lambda \rightarrow \widehat{\Delta}(\mathfrak{a})$ be a mapping defined 
by

$$
\widehat{\phi}(\lambda)= \begin{cases}\phi(\lambda) & \text { if } \lambda \neq \mu_{\Lambda}, \\ \mu & \text { if } \lambda=\mu_{\Lambda} .\end{cases}
$$

The above ordered set embedding yields the $o$-group embedding of $V(\Lambda, \mathbb{R})$ into $V(\widehat{\Delta}(\mathfrak{a}), \mathbb{R})$ by $\left(\ldots, f_{\lambda}, \ldots\right) \mapsto\left(\ldots, f_{\pi}, \ldots\right)$, where $\pi \in \widehat{\Delta}(\mathfrak{a})$ and

$$
f_{\pi}= \begin{cases}f_{\widehat{\phi}(\lambda)} & \text { if } \pi \in \widehat{\phi}(\Lambda), \\ 0 & \text { if } \pi \notin \widehat{\phi}(\Lambda) .\end{cases}
$$

It is clear that $\mathbf{1}(\Lambda)$ is mapped to $\mathbf{1}(\widehat{\Delta}(\mathfrak{a}))$.

ThEOREM 2.4. For a given cardinal number $\mathfrak{a}$ there exists a totally ordered $M V$-algebra $M(\mathfrak{a})$ such that every totally ordered $M V$-algebra of cardinality at most $\mathfrak{a}$ embeds in $M(\mathfrak{a})$.

Proof. Let $A$ be a totally ordered MV-algebra of cardinality $\mathfrak{a}$. If $\mathfrak{a}<\aleph_{0}$, then for some $n, A$ is isomorphic to $\mathrm{E}_{n}$, the $n$-element Łukasiewicz chain, and thus embeds into $\Gamma(\mathbb{Q}, 1)$. So in this case we put $M(\mathfrak{a})=\Gamma(\mathbb{Q}, 1)$. For $\mathfrak{a} \geq \aleph_{0}$, by the Mundici Equivalence Theorem, there is an abelian $o$-group $G$ with a strong order unit $u$ such that $A$ is isomorphic to $\Gamma(G, u)$. As sets, $A=[0, u]$ and $G^{+}=\bigcup_{n=1}^{\infty}[0, n u]=\bigcup_{n=0}^{\infty}[n u,(n+1) u]$, and for every $n=0,1, \ldots$, $|[n u,(n+1) u]|=|[0, u]|=|A|=\mathfrak{a}$, and since $\mathfrak{a}$ is infinite, $|G|=\left|G^{+}\right|=\mathfrak{a}$. By Corollary 2.2, $G$ embeds into $V(\Lambda, \mathbb{R})$ for some totally ordered $\Lambda$ of cardinality $\mathfrak{a}$, with a maximal element and such that $u$ is mapped to $\mathbf{1}(\Lambda)$. Then by Lemma 2.3 , it further embeds into $V(\widehat{\Delta}(\mathfrak{a}), \mathbb{R})$ and $u$ is mapped to $\mathbf{1}(\widehat{\Delta}(\mathfrak{a}))$. Therefore we put $M(\mathfrak{a})=\Gamma(V(\widehat{\Delta}(\mathfrak{a}), \mathbb{R}), \mathbf{1}(\widehat{\Delta}(\mathfrak{a})))$.

If $\mathfrak{a}=\aleph_{0}$, the construction of the target MV-algebra becomes much simpler due to the fact that all countable totally ordered sets order-embed into the rationals. Let $\widehat{\mathbb{Q}}=\mathbb{Q} \cup\{\infty\}$ denote the usually ordered set of the rationals extended by the maximal element $\infty$.

THEOREM 2.5. Every at most countable totally ordered MV-algebra embeds into $\Gamma(V(\widehat{\mathbb{Q}}, \mathbb{R}), \mathbf{1}(\widehat{\mathbb{Q}}))$.

Proof. Since every countable totally ordered set order-embeds into the usually ordered $\mathbb{Q}$ (see e.g. [3, Chapter 3, Theorem 1$]$ ), instead of $\widehat{\Delta}\left(\aleph_{0}\right)$ in the proof of Theorem 2.4, we simply take $\widehat{\mathbb{Q}}$ and the result follows.

Let us agree in this case that $M\left(\aleph_{0}\right)=\Gamma(V(\widehat{\mathbb{Q}}, \mathbb{R}), \mathbf{1}(\widehat{\mathbb{Q}}))$.

COROLlary 2.6. Every subdirectly irreducible MV-algebra of cardinality at most $\aleph_{0}$ embeds into $\Gamma(V(\widehat{\mathbb{Q}}, \mathbb{R}), \mathbf{1}(\widehat{\mathbb{Q}}))$.

Proof. The natural order of a subdirectly irreducible MV-algebra is total, so the result follows by Theorem 2.5. 
We summarize the MV-algebra embeddings in the following:

COROLlary 2.7. Let $\mathfrak{a}$ be any nonzero cardinal number, and let

$$
M(\mathfrak{a})= \begin{cases}\Gamma(V(\widehat{\Delta}(\mathfrak{a}), \mathbb{R}), \mathbf{1}(\widehat{\Delta}(\mathfrak{a}))) & \text { if } \mathfrak{a}>\aleph_{0}, \\ \Gamma(V(\widehat{\mathbb{Q}}, \mathbb{R}), \mathbf{1}(\widehat{\mathbb{Q}})) & \text { if } \mathfrak{a}=\aleph_{0}, \\ \Gamma(\mathbb{Q}, 1) & \text { if } \mathfrak{a}<\aleph_{0} .\end{cases}
$$

Then every totally ordered $M V$-algebra of cardinality less than or equal to $\mathfrak{a}$ embeds in $M(\mathfrak{a})$.

3. Remarks. In the 1930's, Jan Łukasiewicz proposed a deductive system for what is now called the infinitely-valued Łukasiewicz propositional logic and conjectured that his infinitely-valued logic is complete for the deductive system he proposed. The deductive system had four axioms and the Modus Ponens rule as the only inference rule for inferring theses from the four axioms (see [12], [4], or [6]). Conjecturing completeness, Łukasiewicz meant that the set of tautologies of his logic coincides with the set of theses that can be inferred within his deductive system. The conjecture was proved by Mordechaj Wajsberg in the 1930's but was never published (see [12]). The first published proof of Eukasiewicz's conjecture is contained in [11]. The first algebraic proof of Łukasiewicz's conjecture is given in [5]. The concept of an MV-algebra has been introduced in [4].

For a set $\Phi \cup\{\alpha\}$ of propositional formulas of Łukasiewicz's logic let $\Phi \vdash_{\infty} \alpha$ mean that $\alpha$ has a proof within Eukasiewicz's deductive system from the set of hypotheses $\Phi$. Next, for an MV-algebra $A$ let $\Phi \models_{A} \alpha$ mean that under every assignment of the elements of $A$ for the propositional letters of $\Phi \cup\{\alpha\}, \alpha$ is valid in $A$ whenever all formulas of $\Phi$ are valid in $A$ under that assignment. To be "valid" in $A$ means to be equal to the greatest element of $A$ in its natural order.

In terms of $\vdash_{\infty}$ and $\models_{A}$, Eukasiewicz's conjecture means that, for every $\alpha$, $\emptyset \vdash_{\infty} \alpha$ iff $\emptyset=_{R} \alpha$, where $R$ is the MV-algebra defined on the interval [0,1] of real numbers. It is worth mentioning (see [13] or [14]) that, for every finite $\Phi$ and $\alpha, \Phi \vdash_{\infty} \alpha$ iff $\Phi \models_{R} \alpha$ and that the equivalence is not true if $\Phi$ is infinite. Every algebra $M(\mathfrak{a})$ we have constructed, however, has the property that, for all $\Phi$ and $\alpha, \Phi \vdash_{\infty} \alpha$ iff $\Phi \models_{M(\mathfrak{a})} \alpha$. In this situation we say that Eukasiewicz's deductive system is strongly complete with respect to the algebra $M(\mathfrak{a})$. The equivalence follows from the known fact that Eukasiewicz's deductive system is algebraizable in the sense of [2] and the fact due to Theorem 2.6 which says that every countable and subdirectly irreducible MV-algebra is embeddable into $M(\mathfrak{a})$; see [9] for a general discussion of this issue.

The algebra $M\left(\aleph_{0}\right)$ with respect to which Łukasiewicz's deductive system is strongly complete is the smallest we currently know. For many important 
nonclassical deductive systems it is known that strongly complete models have to be uncountable (see [8], [15]). It has been pointed out and proven by the referee of this paper that also in our case, the algebra $M\left(\aleph_{0}\right)$ must be uncountable. We include the proof of this important observation below.

Theorem 3.1. An MV-algebra A with the property that every totally ordered countable $M V$-algebra embeds into $A$ is uncountable.

Proof. Using the unital $\ell$-group equivalence again, suppose that $G$ is an abelian $o$-group with a strong order unit $u$ into which every countable unital $o$-group can be embedded. Let $C$ be the maximal proper convex subgroup of $G$. Then $G / C$ is an o-group without any proper convex subgroups and thus it is archimedean, and consequently by Hölder's Theorem, $G / C$ is isomorphic to a subgroup of the real numbers ([1, Theorem 2.3]). By Corollary 2.2 we can assume that $u$ corresponds to 1 , so that if $\pi: G \rightarrow G / C$ is the natural homomorphism, then we have $\pi: G \rightarrow \mathbb{R}$ and $\pi(u)=1$.

Consider any unital o-group of the form $\mathbb{Q}+\mathbb{Q} \alpha$ where $\alpha \in \mathbb{R} \backslash \mathbb{Q}$, and the unit is 1 . Then we have the embedding: $\phi: \mathbb{Q}+\mathbb{Q} \alpha \hookrightarrow G$ and $\phi(1)=u$. Next consider the homomorphism $\pi \phi: \mathbb{Q}+\mathbb{Q} \alpha \rightarrow \mathbb{R}$. It is an injection since $\mathbb{Q}+\mathbb{Q} \alpha$ is archimedean. We have $\pi \phi(1)=\pi(u)=1$, so $\pi \phi$ is the identity on $\mathbb{Q}$, which is dense in $\mathbb{R}$, therefore $\pi \phi$ is the identity on the entire $\mathbb{Q}+\mathbb{Q} \alpha$. Since $\mathbb{R}$ is the union of groups of this form, it follows that there is an embedding of $\mathbb{R}$ into $G / C$. Thus we have $\mathbb{R} \hookrightarrow G / C \subseteq \mathbb{R}$, hence $G / C=\mathbb{R}$. Therefore, $|G| \geq|\mathbb{R}|>\aleph_{0}$.

Acknowledgements. The author is very grateful to Professors Wiesław Dziobiak and W. Charles Holland for their encouragement and help, and to the referee for the solution of the cardinality problem in Theorem 3.1 and other improvements of the paper.

\section{References}

[1] M. Anderson and T. Feil Lattice-Ordred Groups, Reidel, 1988.

[2] W. J. Blok and D. Pigozzi, Algebraizable logics, Mem. Amer. Math. Soc. 77 (1989), no. 396 .

[3] G. Birkhoff, Lattice Theory, Amer. Math. Soc., 1948.

[4] C. C. Chang, Algebraic analysis of many-valued logics, Trans. Amer. Math. Soc. 88 (1958), 476-490.

[5] -, A new proof of the completeness of the Eukasiewicz axioms, ibid. 93 (1959), $74-80$.

[6] R. Cignoli, I. M. D'Ottaviano and D. Mundici, Algebraic Foundations of ManyValued Logic, Kluwer, Dordrecht, 2000.

[7] P. Conrad, The group of order preserving automorphisms of an ordered abelian group, Proc. Amer. Math. Soc. 9 (1958), 382-389.

[8] W. Dziobiak, Nonexistence of a countable strongly adequate matrix semantics for neighbours of E, Bull. Sect. Logic Polish Acad. Sci. 10 (1981), 170-176. 
[9] W. Dziobiak, A. V. Kravchenko, and P. Wojciechowski, Equivalents for a quasivariety to be generated by a single algebraic structure, Studia Logica 91 (2009), 113-123.

[10] D. Mundici, Interpretation of $A F C^{*}$-algebras in Lukasiewicz sentential calculus, J. Funct. Anal. 65 (1986), 15-63.

[11] A. Rose and J. B. Rosser, Fragments of many-valued statement calculi, Trans. Amer. Math. Soc. 87 (1958), 1-53.

[12] A. Tarski, Logic, Semantics, and Metamathematics, Oxford Univ. Press, 1956.

[13] R. Wójcicki, On matrix representation of consequence operations on Eukasiewicz's sentential calculi, Z. Math. Logik Grundlagen Math. 19 (1976), 239-247.

[14] - Theory of Logical Calculi: Basic Theory of Consequence Operations, Kluwer, 1988.

[15] A. Wroński, On cardinalities of matrices adequate for the intuitionistic propositional logic, Rep. Math. Logic 3 (1974), 67-72.

Department of Mathematical Sciences

The University of Texas at El Paso

El Paso, TX 79968, U.S.A.

E-mail: piotrw@utep.edu

Received 10 September 2008;

in revised form 20 January 2009 